\title{
Quantitative RT-PCR evaluation of a rapid influenza antigen test for efficient diagnosis of influenza virus infection
}

Yuko Tsushima ${ }^{\mathrm{a}}$, Naoki Uno ${ }^{\mathrm{a}, \mathrm{b}, *}$, Daisuke Sasaki ${ }^{\mathrm{a}}$, Yoshitomo Morinaga ${ }^{\mathrm{a}, \mathrm{b}}$, Hiroo Hasegawa $^{\mathrm{a}, \mathrm{b}}$, and Katsunori Yanagihara ${ }^{\mathrm{a}, \mathrm{b}}$

${ }^{a}$ Clinical Laboratory of Nagasaki University Hospital, Nagasaki 852-8501, Japan

${ }^{b}$ Department of Laboratory Medicine, Nagasaki University Graduate School of Biomedical Sciences, Nagasaki 852-8501, Japan

*Corresponding author: Department of Laboratory Medicine, Nagasaki University Graduate School of Biomedical Sciences, 1-7-1 Sakamoto, Nagasaki 852-8501, Japan Tel.: +81 95819 7574; Fax: +81 958197422

E-mail:nuno@nagasaki-u.ac.jp (N.Uno)

1

\footnotetext{
1 Abbreviations: RIDT: rapid influenza antigen diagnostic test; NAAT: nucleic acid amplification test; qRT-PCR: quantitative reverse transcription-polymerase chain reaction.
} 


\begin{abstract}
Influenza virus infection is diagnosed in most cases using a rapid influenza antigen diagnostic test (RIDT). However, false-negative results are a major concern. By contrast, the nucleic acid amplification test offers high sensitivity and therefore can aid the interpretation of negative RIDT results. In this study, influenza viral loads were quantified with quantitative reverse transcription-polymerase chain reaction (qRT-PCR) using viral suspensions left over after RIDT, and the performance of both methods was evaluated. qRT-PCR detected as few as $10^{3}$ copies/mL of influenza viruses A and B, whereas RIDT showed negative results for viral loads less than $10^{7}$ and $10^{5}$ copies $/ \mathrm{mL}$ of influenza viruses A and B, respectively. These results indicate that small quantities of the virus that cause false-negative RIDT results can be detected efficiently with qRT-PCR follow-up. In addition, influenza A virus subtype was determined using qRT-PCR.
\end{abstract}

\title{
Keywords
}

Influenza virus, Rapid influenza antigen detection test, quantitative RT-PCR 


\section{Introduction}

The rapid influenza antigen diagnostic test (RIDT) using immunochromatography is used widely to detect viral antigens. RIDT requires no special skills or instruments and has a short turnaround time; therefore, it is a common point-of-care test for the detection of influenza virus infections (Welch and Ginocchio, 2010). Although its usual specificity exceeds $90 \%$, its analytical sensitivity is variable, ranging from $10 \%$ to $80 \%$ (Chartrand et al., 2012; Uyeki et al., 2009). This variability may be attributable to differences in kit contents such as the medium or swab (Hurt et al., 2007; Luinstra et al., 2011; Smieja et al., 2010), patient age (Hurt et al., 2007; Ruest et al., 2003), the type of respiratory specimen (Agoritsas et al., 2006), and the time of sampling from illness onset (Ward et al., 2004). In particular, physical factors during sample collection have direct effects on the results (Smieja et al., 2010).

Conversely, the nucleic acid amplification test (NAAT) offers high sensitivity and it has therefore been developed for the detection of various viruses (Templeton et al., 2004). This technique also detects multiple targets in multiple samples (Wu et al., 2008; Yang et al., 2010). Despite these advantages, NAAT is not in general use in the clinical setting because it is complex and time-consuming. The Centers for Disease Control and Prevention (2013) recommend that further influenza testing be considered for patients who test negative with RIDT when community influenza activity is high and laboratory confirmation of influenza is desirable. However, further testing requires additional sample collection. From a pragmatic point of view, NAAT would be most beneficial if it were performed with the sample material left over after RIDT. Moreover, technical bias from the sample collection process would be eliminated if the same sample was used. In the present study, influenza viral loads were quantified using quantitative reverse transcription-polymerase chain reaction (qRT-PCR) in viral suspensions left over after RIDT, and the performance of both methods was evaluated.

\section{Materials and Methods}

\subsection{Clinical specimens}

Two hundred fifty-five nasopharyngeal swab specimens were collected from patients at Nagasaki University Hospital between December 2012 and March 2013. All clinical specimens were examined using a Clearline Influenza A/B (H1N1) 2009 assay (Alere Medical, Tokyo, Japan) according to the manufacturer's instructions. Viral suspension 
that remained after RIDT was stored at $-80^{\circ} \mathrm{C}$ until RNA extraction.

\subsection{Viral RNA preparation}

Viral RNA was extracted directly from a $140-\mu \mathrm{L}$ viral suspension using a QIAamp Viral RNA Mini kit (QIAGEN, Hilden, Germany) and digested with Amplification Grade DNase I (Sigma Aldrich, St. Louis, MO, USA) according to the manufacturer's instructions. Subsequently, the viral RNA was diluted with four volumes of RNase-free water and used as the template for qRT-PCR. For the positive control, AMPLIRUN Influenza A H1, H3, and B RNA controls were purchased from Vircell Microbiologists (Granada, Spain).

\subsection{Primer and probe design for $q R T-P C R$}

The primers used for influenza A virus quantification were modified partially from those described previously (Centre for Health Protection, 2009; Ward et al., 2004), and the probe was selected from the Universal Probe Library (Roche Applied Science, Mannheim, Germany). The primers and probes used for influenza A virus subtyping (Centre for Health Protection, 2009) and influenza B virus quantification were as described previously (van Elden et al., 2001; Wu et al., 2008), with modifications.

\subsection{Preparation of viral RNA standards for viral load quantification}

Complementary DNA was synthesized from AMPLIRUN Influenza A H3 and B RNA controls using SuperScript III Reverse Transcriptase (Invitrogen, Carlsbad, CA, USA) and random primers (Invitrogen). PCR was performed with AmpliTaq Gold (Applied Biosystems, Foster City, CA, USA) to amplify a matrix gene of the influenza A virus and a hemagglutinin gene of the influenza B virus using primers FLUAM-1F/1R and INFB-1/2, respectively (Table 1 ). The PCR products were isolated and purified using a QIAquick PCR purification kit (QIAGEN) before cloning with a TA PCR Cloning kit (pTAC-1; Bio Dynamics Laboratory, Tokyo, Japan). Plasmids were recovered using a QIAprep Spin Miniprep kit (QIAGEN) and sequenced with a 3130 Genetic Analyzer (Applied Biosystems). The plasmid of influenza A was amplified further with PCR using primers FLUAM-1R and M13-T7_rev (5'-TTCTAATACGACTCACTATAGGGCGGATAACAATTTCACACAG-3'). The PCR product was purified with the QIAquick PCR purification kit and used as the template 
for in vitro transcription. The plasmid of influenza B was digested by the restriction enzyme BamHI (Takara Bio, Shiga, Japan) and purified using the QIAquick PCR purification kit. RNA was synthesized via in vitro transcription using a MEGAshortscript T7 kit (Ambion, Austin, TX, USA) and purified using a MEGAclear kit (Ambion). Purified RNA was separated via MultiNA capillary electrophoresis (Shimadzu, Kyoto, Japan), and a single band was confirmed.

\section{5. $q R T-P C R$}

One-step RT-PCR was performed using LightCycler 480 RNA Master Hydrolysis Probes (Roche Applied Science) in $20-\mu \mathrm{L}$ reaction mixtures containing $5 \mu \mathrm{L}$ diluted viral RNA, 7.3 $\mu$ L LightCycler 480 RNA Master Hydrolysis Probes (2.7 × conc.), 1.3 $\mu \mathrm{L}$ Activator, $1 \mu \mathrm{L}$ Enhancer $(20 \times$ conc.), $0.5 \mu \mathrm{M}$ primer (each), and $0.25 \mu \mathrm{M}$ probe (each). The primers and probes used are shown in Table 1. RT-PCR conditions were $63^{\circ} \mathrm{C}$ for $3 \mathrm{~min}$ and $95^{\circ} \mathrm{C}$ for $30 \mathrm{~s}$, followed by 45 cycles of $95^{\circ} \mathrm{C}$ for $10 \mathrm{~s}$ and $58^{\circ} \mathrm{C}$ for 30 s. Standard curves were drawn from serial dilutions of viral RNA standards. For positive RIDT samples, either the influenza A or the influenza B virus was quantified. For negative RIDT samples, the influenza A and B viruses were quantified separately. qRT-PCR was performed using both $\mathrm{H} 1$ and $\mathrm{H} 3$ primers for positive RIDT samples to subtype the influenza A virus biplex. For negative RIDT samples, qRT-PCR was performed separately for $\mathrm{H} 1$ and $\mathrm{H} 3$ subtyping because the biplex reaction was found to be less sensitive than the monoplex reaction.

\section{Results}

\subsection{Comparison of RIDT and qRT-PCR results}

RIDT showed a positive result in 34 of 255 samples, which included 31 seasonal influenza A and 3 influenza B infections (Fig. 1). Pandemic influenza A 2009 (H1N1) was not detected in any sample. qRT-PCR was performed using these 34 positive RIDT samples and 77 randomly selected negative RIDT samples obtained from 101 patients. The median age of the patients was 42 years (range, 0-92 years). All patients, except two, had fever, respiratory symptoms, or both. The median time from illness onset to specimen collection was 1 day (range, 0 to $>7$ days). The two patients without fever and respiratory signs lived with persons infected with influenza virus. Viral RNA was readily amplified in all of the positive RIDT samples in concordance with the type of 
influenza virus. For the 77 negative RIDT samples, amplification was observed clearly in 22 samples for influenza A, three for influenza B, and one for both influenza types, whereas 51 samples lacked amplification for either influenza A or B (Fig. 1).

\subsection{Limits of detection of RIDT and $q R T-P C R$}

Viral copy number was assessed in 34 positive and 26 negative RIDT samples that were determined to be positive using qRT-PCR. The quantum of the influenza A viral load ranged from $10^{7}$ to $10^{11}$ copies $/ \mathrm{mL}$ in the positive RIDT samples and $10^{3}$ to $10^{8}$ copies/mL in the negative RIDT samples (Fig. 2). The quantum of the influenza B virus ranged from $10^{5}$ to $10^{9}$ copies/mL in the positive RIDT samples and $10^{3}$ to $10^{5}$ copies/mL in the negative RIDT samples (see Fig. 2). Accordingly, the limit of detection of RIDT was $10^{7}$ to $10^{8}$ copies/mL for influenza A and $10^{5}$ copies/mL for influenza $B$, whereas that of qRT-PCR was $10^{3}$ copies/mL for both influenza A and B. These outcomes indicate that low copy numbers of the viruses that result in negative RIDT results can be detected with qRT-PCR.

\subsection{Influenza A virus subtyping}

Influenza A subtype was determined using subtype-specific primers and probes for 31 influenza A samples detected by RIDT and 23 influenza A samples detected by qRT-PCR but not RIDT. The 31 samples detected by RIDT were amplified clearly by H3 primers. In the 23 samples detected by only qRT-PCR, eight samples were amplified by H3 primers and 15 samples were amplified by neither H3 nor H1 primers, perhaps due to the low viral copy number. Therefore, all influenza A viruses amplified by subtype-specific primers were of the H3 subtype.

\subsection{Clinical outcome of patients with false-negative RIDT results}

The clinical outcome of the 24 patients with positive qRT-PCR but negative RIDT results was assessed retrospectively. All of the patients except one received no antiviral agents, whereas 20 patients were prescribed antibiotics. During antibiotic therapy, a patient showed serious influenza symptoms for more than 8 days in the absence of a clear diagnosis. Another patient was admitted after antibiotic therapy with a diagnosis of pneumonia and was further treated with another antibiotic. Some of the other patients received multiple examinations to determine causes for their illness other than influenza 
virus, but ultimately, the results did not account for their symptoms.

\section{Discussion}

Despite inappropriate treatment in patients with positive qRT-PCR results but negative RIDT results, all patients who did not receive antivirals recovered eventually from their influenza symptoms. However, the illnesses of two patients progressed to pneumonia with hypoxia. One developed pneumonia after steroid pulse therapy for myelitis. RIDT was performed 4 days after fever onset and gave a negative result, whereas qRT-PCR detected $1.3 \times 10^{5}$ copies/mL of influenza A virus in the same specimen. Bronchoscopy and blood tests performed to obtain causal information did not result in a definitive diagnosis. This patient also presented with encephalopathy, although its relevance to the influenza virus was unclear. The other patient developed pneumonia and serious hypoxia with pulmonary oedema due to chronic renal failure. RIDT was performed once on days 1 and 2 after symptom onset, but the results for both specimens were negative, whereas qRT-PCR detected $1.24 \times 10^{5}$ copies/mL of influenza B virus in the second specimen. These results indicate that infection with even a small amount of influenza virus can have serious outcomes if patients are at risk for complications such as immunosuppressive therapy or chronic renal dysfunction.

The RIDT results were obtained using one commercial kit in this study. However, the stability of viral RNA in the solution included with RIDT kits was evaluated with qRT-PCR using two RIDT kits: Clearline Influenza A/B and QuickNavi-Flu (Denka Seiken, Niigata, Japan). A clinical viral sample was placed in the solution provided by each kit and stored under various conditions to select a kit and determine a storage condition for the experiments. The qRT-PCR results showed that viral RNA was sufficiently amplified without remarkable degradation when samples were stored at $-80^{\circ} \mathrm{C}$, and no substantial differences were found between the two kits (data not shown). These results suggest that the kit used in the experiments was representative of other commercially available RIDT kits.

The present study provides evidence that influenza infection cannot be ruled out by negative RIDT results because the test has low sensitivity. Particularly when influenza activity is high, the negative predictive value of RIDT is low, and therefore the chance that a negative result is a true negative is also low (Harper et al., 2009). Nevertheless, antiviral treatment was not initiated for most patients who tested negative 
with RIDT but positive with qRT-PCR, indicating that the RIDT results affected clinical decision-making to a considerable extent. In other words, most clinicians ruled out influenza virus infection based on negative RIDT results despite the high rate of false-negative results with RIDT. Such judgments might lead to inappropriate antibiotic use, unnecessary examinations, and further viral spread. NAAT can be performed when RIDT results are inconsistent with clinical presentation. However, as a general rule, NAAT requires additional sample collection. The results of the present study show that samples left over after RIDT can be used for NAAT and that even a quantity of virus as low as $10^{3}$ copies/mL can be detected using qRT-PCR without invasive sample re-collection. Furthermore, qRT-PCR allows the determination of influenza A subtype. Taken together, these results suggest that effective and accurate clinical decision-making may be facilitated by the implementation of NAAT using samples left over after RIDT.

\section{Acknowledgements}

We thank the staff of the clinical microbiology laboratory of Nagasaki University Hospital for sample collection.

\section{References}

Agoritsas, K., Mack, K., Bonsu, B.K., Goodman, D., Salamon, D., Marcon, M.J., 2006. Evaluation of the Quidel QuickVue test for detection of influenza A and B viruses in the pediatric emergency medicine setting by use of three specimen collection methods. J. Clin. Microbiol. 44, 2638-2641.

Centers for Disease Control and Prevention, 2013. Guidance for Clinicians on the Use of Rapid Influenza Diagnostic Tests. http://www.cdc.gov/flu/professionals/diagnosis/clinician_guidance_ridt.htm. Accessed October 13, 2014.

Centre for Health Protection, 2009. CHP molecular diagnostic protocols for the detection of human swine influenza virus type A (subtype H1) revision 2.

Chartrand, C., Leeflang, M.M., Minion, J., Brewer, T., Pai, M., 2012. Accuracy of rapid influenza diagnostic tests: a meta-analysis. Ann. Intern. Med. 156, 500-511.

Harper, S.A., Bradley, J.S., Englund, J.A., File, T.M., Gravenstein, S., Hayden, F.G., McGeer, A.J., Neuzil, K.M., Pavia, A.T., Tapper, M.L., Uyeki, T.M., 
Zimmerman, R.K., 2009. Seasonal influenza in adults and children-diagnosis, treatment, chemoprophylaxis, and institutional outbreak management: clinical practice guidelines of the Infectious Diseases Society of America. Clin. Infect. Dis. 48, 1003-1032.

Hurt, A.C., Alexander, R., Hibbert, J., Deed, N., Barr, I.G., 2007. Performance of six influenza rapid tests in detecting human influenza in clinical specimens. J. Clin. Virol. 39, 132-135.

Luinstra, K., Petrich, A., Castriciano, S., Ackerman, M., Chong, S., Carruthers, S., Ammons, B., Mahony, J.B., Smieja, M., 2011. Evaluation and clinical validation of an alcohol-based transport medium for preservation and inactivation of respiratory viruses. J. Clin. Microbiol. 49, 2138-2142.

Ruest, A., Michaud, S., Deslandes, S., Frost, E.H., 2003. Comparison of the Directigen flu A+B test, the QuickVue influenza test, and clinical case definition to viral culture and reverse transcription-PCR for rapid diagnosis of influenza virus infection. J. Clin. Microbiol. 41, 3487-3493.

Smieja, M., Castriciano, S., Carruthers, S., So, G., Chong, S., Luinstra, K., Mahony, J.B., Petrich, A., Chernesky, M., Savarese, M., Triva, D., 2010. Development and evaluation of a flocked nasal midturbinate swab for self-collection in respiratory virus infection diagnostic testing. J. Clin. Microbiol. 48, 3340-3342.

Templeton, K.E., Scheltinga, S.A., Beersma, M.F., Kroes, A.C., Claas, E.C., 2004. Rapid and sensitive method using multiplex real-time PCR for diagnosis of infections by influenza A and influenza B viruses, respiratory syncytial virus, and parainfluenza viruses 1, 2, 3, and 4. J. Clin. Microbiol. 42, 1564-1569.

Uyeki, T.M., Prasad, R., Vukotich, C., Stebbins, S., Rinaldo, C.R., Ferng, Y.H., Morse, S.S., Larson, E.L., Aiello, A.E., Davis, B., Monto, A.S., 2009. Low sensitivity of rapid diagnostic test for influenza. Clin. Infect. Dis. 48, e89-92.

van Elden, L.J., Nijhuis, M., Schipper, P., Schuurman, R., van Loon, A.M., 2001. Simultaneous detection of influenza viruses $A$ and $B$ using real-time quantitative PCR. J. Clin. Microbiol. 39, 196-200.

Ward, C.L., Dempsey, M.H., Ring, C.J., Kempson, R.E., Zhang, L., Gor, D., Snowden, B.W., Tisdale, M., 2004. Design and performance testing of quantitative real time PCR assays for influenza A and B viral load measurement. J. Clin. Virol. 29, 179-188. 
Welch, D.F., Ginocchio, C.C., 2010. Role of rapid immunochromatographic antigen testing in diagnosis of influenza A virus 2009 H1N1 infection. J. Clin. Microbiol. 48, 22-25.

Wu, C., Cheng, X., He, J., Lv, X., Wang, J., Deng, R., Long, Q., Wang, X., 2008. A multiplex real-time RT-PCR for detection and identification of influenza virus types A and B and subtypes H5 and N1. J. Virol. Methods 148, 81-88.

Yang, Y., Gonzalez, R., Huang, F., Wang, W., Li, Y., Vernet, G., Wang, J., Jin, Q., 2010. Simultaneous typing and HA/NA subtyping of influenza A and B viruses including the pandemic influenza A/H1N1 2009 by multiplex real-time RT-PCR. J. Virol. Methods 167, 37-44.

\section{Conflict of interest}

None declared.

\section{Figure legend}

Fig. 1. Sequential results of the rapid influenza antigen diagnostic test (RIDT) and quantitative reverse transcription-polymerase chain reaction (qRT-PCR). Influenza A and $\mathrm{B}$ are highlighted in light and dark grey, respectively. *One sample was positive for both influenza A and B.

Fig. 2. Quantification of influenza viruses A and B with quantitative reverse transcription-polymerase chain reaction and the results of the rapid influenza antigen diagnostic test (RIDT). Large quantities of virus were detected in 34 samples with positive RIDT results, whereas smaller quantities were detected in 26 samples with negative RIDT results. 


\section{Table}

Table 1. Primer and probe sequences

\begin{tabular}{|c|c|c|}
\hline $\begin{array}{l}\text { Primer and } \\
\text { probe names }\end{array}$ & Oligonucleotide sequence (5'-3') & Reference \\
\hline \multicolumn{3}{|c|}{ Primers and probe for quantification of the influenza A matrix gene } \\
\hline FLUAM-1F & AAGACCAATYYTGTCACCTCTGA & $\begin{array}{l}\text { Centre for Health Protection, 2009; } \\
\text { Ward et al., 2004, with modifications }\end{array}$ \\
\hline FLUAM-1R & CAAAGCGTCTACGCTGCAGTCC & $\begin{array}{l}\text { Centre for Health Protection, 2009; } \\
\text { Ward et al., } 2004\end{array}$ \\
\hline UPL probe104 & FAM-GTGCCCAG-TAMRA & $\begin{array}{l}\text { Centre for Health Protection, 2009; } \\
\text { Ward et al., } 2004\end{array}$ \\
\hline \multicolumn{3}{|c|}{ Primers and probe for quantification of the influenza B hemagglutinin gene } \\
\hline INFB-1 & AAATACGGTGGATTAAAYAAAAGCAA & $\begin{array}{l}\text { van Elden et al., 2001, with } \\
\text { modifications; Wu et al., } 2008\end{array}$ \\
\hline INFB-2 & CCAGCAATAGCTCCGAAGAAA & van Elden et al., 2001; Wu et al., 2008 \\
\hline INFB probe & $\begin{array}{l}\text { Су5-САСССАTATTGGGCAATTTCCTATGGC } \\
\text {-ВHQ3 }\end{array}$ & van Elden et al., 2001; Wu et al., 2008 \\
\hline \multicolumn{3}{|c|}{ Primers and probe for the influenza A hemagglutinin gene (H1 subtype) } \\
\hline H1-247F & AACATGTTACCCAGGGCATTTCGC & Centre for Health Protection, 2009 \\
\hline H1-361R & GTGGTTGGGCCATGAGCTTTCTTT & Centre for Health Protection, 2009 \\
\hline H1-278P & $\begin{array}{l}\text { Сy5-GAGGAACTGAGGGAGCAATTGAGTTC } \\
\text { AG-BHQ3 }\end{array}$ & $\begin{array}{l}\text { Centre for Health Protection, 2009, } \\
\text { with modifications }\end{array}$ \\
\hline \multicolumn{3}{|c|}{ Primers and probe for the influenza A hemagglutinin gene (H3 subtype) } \\
\hline H3-293f F & ACCCTCAGTGTGATGGCTTCCAAA & Centre for Health Protection, 2009 \\
\hline H3-400R & TAAGGGAGGCATAATCCGGCACAT & Centre for Health Protection, 2009 \\
\hline H3-342P & $\begin{array}{l}\text { HEX-ACGCAGCAAAGCCTACAGCAACTGT } \\
\text { T-BHQ1 }\end{array}$ & Centre for Health Protection, 2009 \\
\hline
\end{tabular}




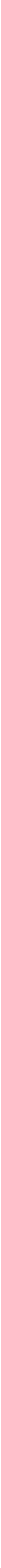




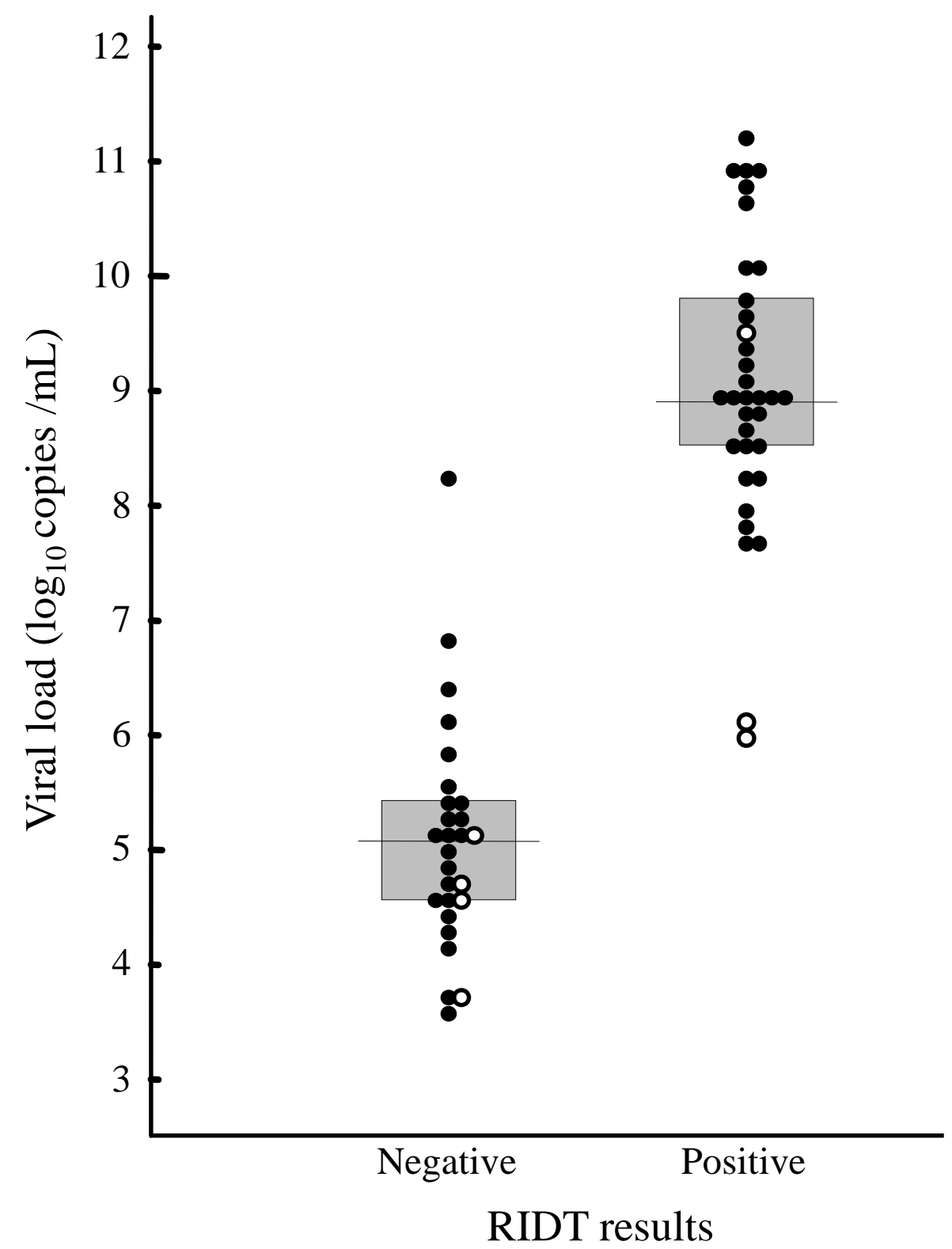

- Influenza A - Influenza B

RIDT results 